\title{
2426. An adaptive stochastic resonance method for weak fault characteristic extraction in planetary gearbox
}

\author{
Zhixing Li ${ }^{1}$, Boqiang Shi ${ }^{2}$ \\ School of Mechanical Engineering, University of Science and Technology Beijing, \\ Beijing, People's Republic of China \\ ${ }^{2}$ Corresponding author \\ E-mail: ${ }^{1}$ onyxlzx@126.com, ${ }^{2}$ shiboqiang@ustb.edu.cn \\ Received 3 September 2016; received in revised form 8 December 2016; accepted 25 December 2016 \\ DOI https://doi.org/10.21595/jve.2016.17652
}

Check for updates

\begin{abstract}
Planetary gearbox is widely used in rotating machinery and prone to damage and failure due to heavy load and complex operating conditions. Furthermore, due to heavy background noise and complicated transmission path, the fault characteristics imbedded in the acquired signals is very weak and difficult to be extracted. Therefore, it is a challenge task for extracting fault characteristics of planetary gearboxes. To tackle this task, this paper proposes an adaptive stochastic resonance method for weak fault characteristic extraction of planetary gearbox, where a chaos ant colony algorithm characterized global optimization ability is employed to achieve adaptive matching between potential parameters and thereby overcoming the local optimization shortcoming of traditional stochastic resonance. Simulation and planetary gearbox experiments with a missing tooth and broken tooth validate the effectiveness of the proposed method, respectively. The results show that the proposed method is not only able to extract weak fault characteristics but also superior to singular value decomposition method.
\end{abstract}

Keywords: planetary gearbox, fault characteristic extraction, adaptive stochastic resonance, chaos ant colony algorithm.

\section{Introduction}

Planetary gearbox has the advantages of compact structure, small volume, strong bearing capacity, high transmission ratio and high transmission efficiency. In hence, it is widely used in areas of aviation engine, metallurgical equipment, vehicles and wind power [1]. However, planetary gearbox operates in bad environment in actual working conditions and bears complex dynamic load force. Such as the transmission mechanism in wind turbine as a power system not only bears variable load but also operating conditions changes frequently. It is the weak link in the entire power transmission part. Once the planetary gearbox has a fault, it may lead to the failure of the entire power system, or even heavy crash. Therefore, planetary gearbox fault diagnosis problem must be taken seriously.

The vibration test is one of the most commonly used method in today's machinery fault diagnosis [2], but planetary gear in planetary gearbox revolves on its own axis while rotation around planet carriers. In Fig. 1, it is a component of planetary gearbox with three planet gears. The vibration signals show the complexity and variability as its own complex motion characteristics and dynamic characteristics. Meanwhile, due to the power input, vibration coupling with each other of the load equipment, modulation on the motion of the planet gear and the interference of background noise, the vibration signal becomes more complicated [3-5]. Thus, the difficulty of signal analysis and fault diagnosis are increased.

To solve this problem, many scholars studied planetary gearbox failure mechanism and proposed a series of effective methods [6]. Samuel et al. [7] proposed a method for gear damage detection of helicopter's planetary gearbox, which based on wavelet lifting of constraint adaptive algorithm. Barszcza et al. [8] applied spectral kurtosis technique to the planetary gearbox detection of wind turbine. Bai et al. [9] proposed a fault diagnosis method combined empirical mode decomposition with support vector machine technology, made full use of the advantages of the two methods. It is used for the early fault diagnosis of the gearbox in the wind turbine. Zhang et 
al. [10] proposed a new denoising method, which formed a collaborative system by blind deconvolution algorithm, fault prediction, vibration model and characteristics extraction, then extracted the vibration signal of helicopter's main gearbox. Hu et al. [11] put forward a quantitative detection method of planetary wheel damage based on grey theory and physical model. Inalpolay et al. [12] proposed a nonlinear dynamic model that was established to study the sidebands structure of the planetary gearbox to extract the fault feature. Feng et al. [13-16] had a great deal of research on planetary gearbox fault and built fault diagnosis model of the planetary gearbox. Thereafter, they studied gear distributed fault and local fault, and made use of ensemble empirical mode decomposition (EEMD) and local mean decomposition to detect the fault of sun gear of planetary gearbox wear and peel off. Mark et al. [17, 18] used modeling studied the spectrum characteristics of the uneven planetary gearbox bearing and the load modulation, and it is expected to be the basis for the fault diagnosis of planetary gearbox.

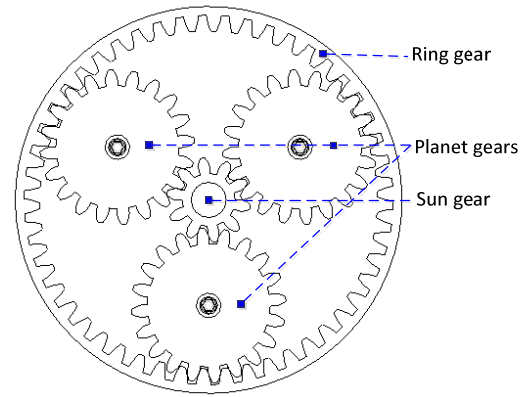

Fig. 1. The planetary gearbox with three planetary gears

The above literature research is based on the noise elimination, but the useful weak signal hidden in the strong background noise is removed by noise elimination at the same time, which has effect on the detection results of the weak fault. In hence, the extraction of the weak characteristic signal of the planetary gearbox has not been solved yet. Stochastic resonance was first proposed during the study on evolution of the ancient meteorological glacier [19]. It is different with the traditional denoising method, on contrary, it utilizes noise energy transfer to weak signal, when strengthen weak fault characteristics accompany with weaken noise. Due to the stochastic resonance has the excellent ability of extracting the weak fault characteristics in the strong background noise, it becomes research hotspots in recent years. Shi et al. [20] used the bee colony algorithm optimize stochastic resonance for multi frequency fault signal. Lei et al. [21] made use of ant colony algorithm optimize stochastic resonance parameters to extract the missing teeth and broken teeth fault in planetary gearbox at different speed. Lu et al. [22, 23] proposed new weak fault signal extraction method to achieve stochastic resonance based on the Wood-Saxon potential function and the full-wave signal construction strategy, the two methods have greatly improved the effect of stochastic resonance. Chen et al. [24] combined EEMD with particle swarm optimization in order to extract the weak fault of planetary gearbox. Yang et al. [25] used artificial fish swarm algorithm in the stochastic parameter optimization to obtain the fault of planetary gearbox, and compare with EEMD to demonstrate that the proposed method has a better effect than EEMD.

Above methods for optimization algorithm of stochastic resonance parameters in the literature are easy to make the parameter optimization process into local extremum. In order to overcome the above shortcomings, this paper makes use of the ergodicity and randomicity of chaotic motion, bases on the solution of ant colony algorithm, and uses chaos algorithm to optimize the neighborhood solution. Firstly, it inherits the superiority of ant colony algorithm in solving optimization problems. Furthermore, it highly overcomes the problems of slow speed and easy to fall into local optimum of ant colony algorithm. Through the simulation and detection of the planetary gearbox test platform, it is effective to diagnose the broken tooth and missing tooth 
faults of the gearbox, and it has obvious advantages compared with the singular value decomposition method.

\section{Stochastic resonance theory}

Stochastic resonance takes advantage of synergistic reaction of the input signal and noise in nonlinear system, transfers part of the noise energy to the useful signal, produces the resonance output and enhance the signal energy, in order to identify the weak signals. The conditions of stochastic resonance are as follows: bistable or multi-stable nonlinear system, the input signal and the noise. Stochastic resonance most obviously strengthened the weak signal when three factors achieve the best match. The commonly stochastic resonance model is a bistable system, which is described by the Langvain equation [26]:

$\frac{d x}{d t}=-U^{\prime}(x)+s(t)+n(t)$.

In Eq. (1), $s(t)$ is the input signal, $n(t)$ is white noise and $n(t)=\sqrt{2 D} \xi(t)$ where $D$ is noise intensity, $\xi(t)$ is Gaussian white noise with the mean value is 0 and variance is $1, x(t)$ is the output signal of the system. The bistable system $U(x)$ output stochastic resonance response by the synergistic action of signal $s(t)$ and noise signal $n(t)$, as shown in Fig. 2.

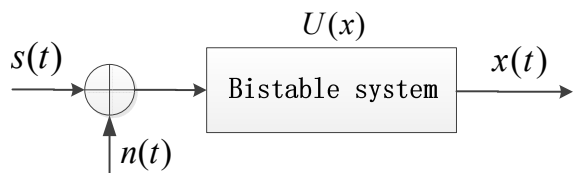

Fig. 2. Bistable stochastic resonance system

The potential function of the bistable stochastic resonance system is defined as:

$U(x)=-\frac{a}{2} x^{2}+\frac{b}{4} x^{4}$

In Eq. (2), it has two stable solution $x= \pm \sqrt{a / b}$ and a non stable solution $x=0$, the barrier height is $\Delta U=a^{2} / 4 b$. It has the minimum potential energy as well as the system at the lowest point of potential well $x= \pm \sqrt{a / b}$ when the external input $s(t)=0$, and the system is most stable at this point. When a weak signal $s(t)$ is inputted into the system, the energy of the signal cannot conquer the obstruct of potential barrier $\Delta U$, so the output state of the system can only move in a potential well; if adds noise $n(t)$ to the system, the input signal becomes $s(t)+n(t)$ and part of the noise energy will convert the signal to engender interaction to overcome the system barrier, so he signal frequency transform between two steady states. Owing to the potential difference of bistable states is much greater than the amplitude of the input signal, the amplitude of the output signal is greater than that of the input signal, so the phenomenon of stochastic resonance occurs.

\section{Adaptive stochastic resonance}

The selection of stochastic resonance system parameters has an important effect on its performance, However, the ant colony algorithm initialize the parameters of stochastic resonance, the pheromone of each path is equal, the pheromone formula is:

$\tau_{i j}(t+1)=(1-\rho) \tau_{i j}(t)+\Delta \tau_{i j}(t)$, 
where $\tau_{i j}(t)$ represents the amount of pheromone on branch $j$ at time $t$, while $t=0$ the amount of pheromone on each path is equal. $\rho$ is the volatile factor of the global pheromone with the general value $[0,1]$, which determines the speed of pheromone volatilization. Ants selects the path according to the same probability and the optimization is difficult, convergence rate is a bit slow. In this paper, chaos ant colony algorithm optimization method of stochastic resonance system parameters is proposed, the ergodicity of chaotic motion is used for chaos initialization and typical chaotic systems logistics mapping as chaos variables is selected. The iterative formula is defined as follows:

$Z_{i j}(t+1)=\mu Z_{i j}(t)\left[1-Z_{i j}(t)\right]$

where $\mu$ is the control parameters with the value [3.56,4], if $\mu=4$ and $0<Z_{i j}<1$, logistics mapping is totally in chaotic state. In order to improve the convergence speed, set a threshold in advance to avoid bad solutions caused the invalid search by after the ants travel once a time. At the same time, chaos disturbance is introduced in adjusting pheromone for avoiding ant colony algorithm falling into local optimization, so it jumps out of local optimum and the improved path of pheromone adjustment is defined as:

$\tau_{i j}(t+1)=(1-\rho) \tau_{i j}(t)+\Delta \tau_{i j}(t)+q_{1} Z_{i j}(t)$.

In Eq. (5), $Z_{i j}(t)$ is chaotic variable which comes from iteration by Eq. (4), $q_{1}$ is coefficient.

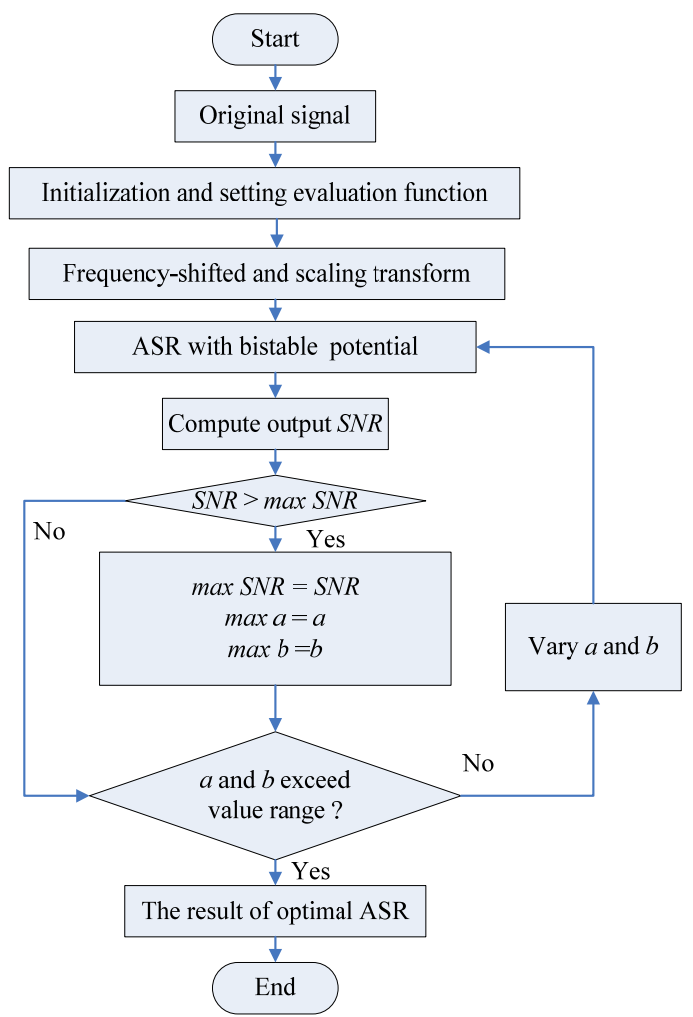

Fig. 3. Flow chart of ASR

In this method, the signal to noise ratio of the stochastic resonance output signal is taken as the evaluation function of the chaotic ant colony optimization process, which reflects the degree of the output signal. According to the requirement of the theory of stochastic resonance, the input 
signal must meet the requirements of small parameters (amplitude $A \ll 1$, noise intensity $D \ll 1$, signal frequency $f \ll 1)$. Therefore, the input signals do not meet the small parameter requirements that must processed by frequency-shifted and scaling transform before the optimization by chaos ant colony algorithm. Thereafter, weak fault features in strong background noise are extracted by adaptive stochastic resonance (ASR). The specific process shows in Fig. 3:

\section{Simulation experiment}

In order to verify the effectiveness of proposed method, simulate a periodic signal [27] with the sampling frequency is $10000 \mathrm{~Hz}$, the characteristic frequency is $100 \mathrm{~Hz}$, the sampling time is $0.3 \mathrm{~s}$, as shown in Fig. 4(a). Furthermore, Gaussian white noise is added to periodic signal for simulating the gear fault signal in strong noise background, which is shown in Fig. 4(b).
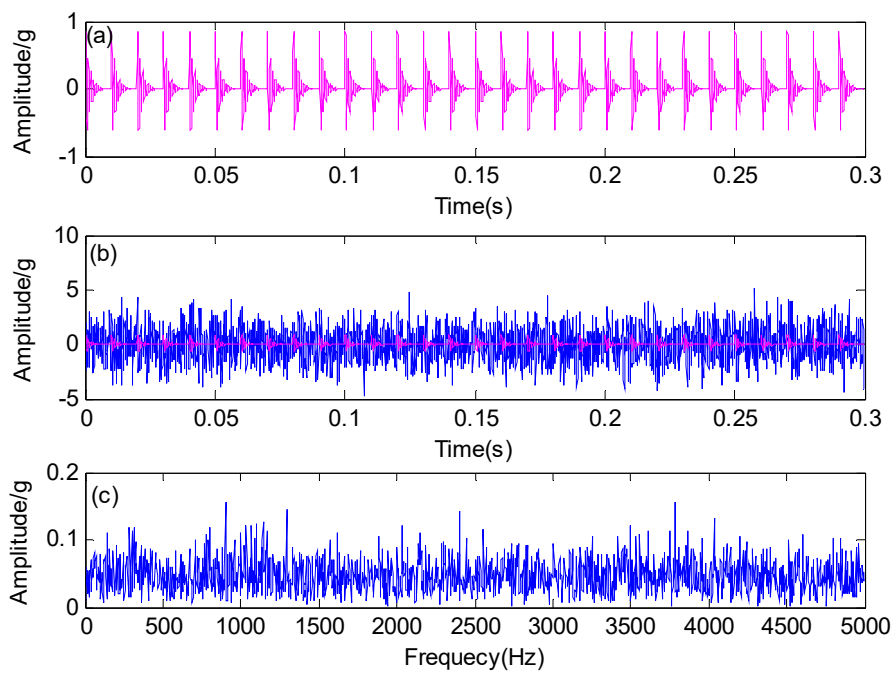

Fig. 4. The simulated fault signal: a) time domain waveform of impulse signal, b) time domain waveform of simulated fault signal for $D=0.5, \mathrm{c}$ ) spectrum of simulated fault signal
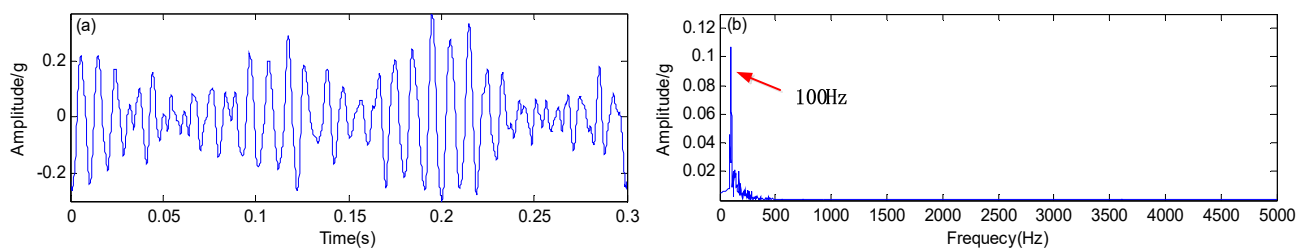

Fig. 5. The ASR method: a) time domain waveform, b) spectrum

Because the periodic signal is submerged in the strong background noise, the characteristic frequency of the periodic signal cannot be obtained as shown in Fig. 4(c). In order to extract the fault features of the simulated signal, in Fig. 5, it illustrates the results by using the ASR method. In Fig. 5(a), the signal is processing by proposed method and shows good periodicity. Besides, in Fig. 5(b), characteristic frequency of $100 \mathrm{~Hz}$ is obviously displayed with the amplitude of 0.1069 , the peak is much higher than ambient noise, and the effectiveness of the proposed method is verified by simulation.

The singular value decomposition method is used for comparison. The method is good at signal processing of fault diagnosis and often used in the detection of weak fault signal, especially the weak periodic signals [28]. Notice that in Fig. 6(b), the noise amplitude value is much higher than the target frequency and the target frequency $100 \mathrm{~Hz}$ is completely hidden by the background 
noise. The results show that singular value decomposition cannot extract characteristic frequency, in other words, the proposed method has obvious superiority to the singular value decomposition method.
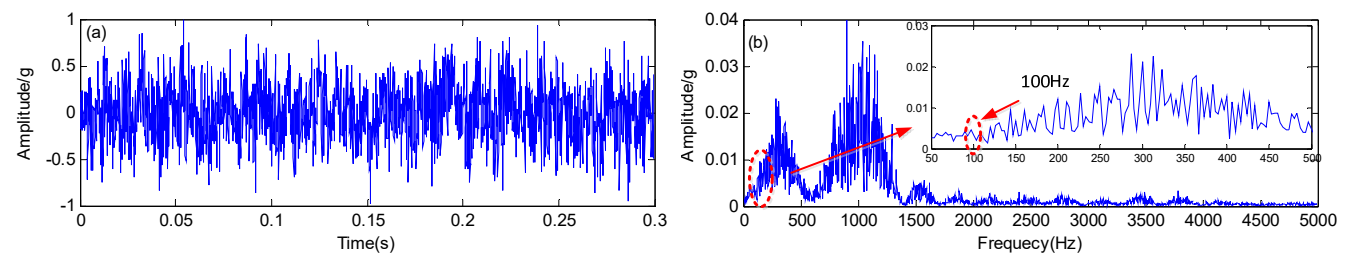

Fig. 6. The singular value decomposition method:

a) time domain waveform, b) spectrum and zoomed-in spectra

\section{Planetary gearbox experimental}

\subsection{Planetary gearbox experimental platform}

In order to verify the effectiveness of proposed method, planetary gearbox is applied in fault diagnosis experiment. In Fig. 7, the devices produced in the United States is used as the experimental platform. Fig. 7(a) is the three-dimensional model and Fig. 7(b) is the physical map of experimental platform. The dynamic system of experimental platform composed with a two-stage planetary gearbox, a fixed-axis gearbox, and driving motor and a programmable magnetic brake. The two-stage planetary gearbox mainly consists of a component with three planetary gears and another component with four planetary gears, and the acceleration sensor is installed on it for measuring vibration signal. The sun gear and a number of planetary gears meshing with each other that prone to abrasion in the actual operation process of the planetary gearbox. Hence, in this experiment, the normal, missing tooth, broken tooth vibration signals of the first stage planetary gearbox are extracted respectively. The physical map of missing tooth, broken tooth of the sun gear is shown in Fig. 8(a) and (b). The parameters of the planetary gearbox and the characteristic frequency of the first stage planetary gearbox are shown in Tables 1 and 2.
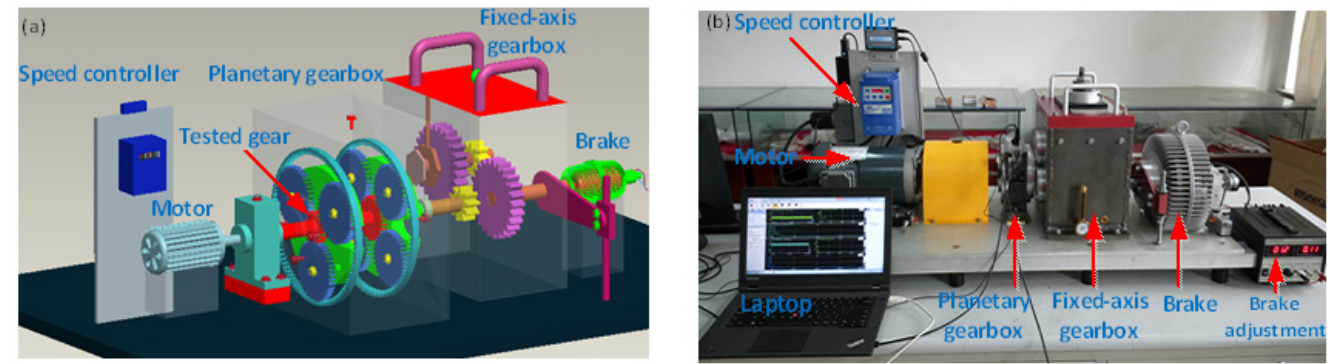

Fig. 7. The experimental platform of planetary gearbox: a) three-dimensional model, b) physical map
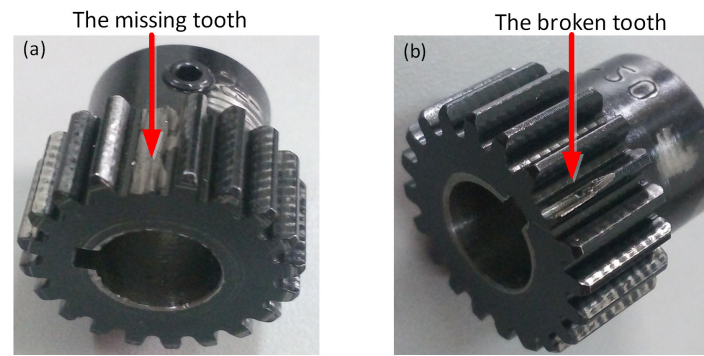

Fig. 8. The physical map of experimental gears: a) missing tooth, b) broken tooth 
Table 1. Parameters of planetary gearbox

\begin{tabular}{|c|c|c|c|c|}
\hline Forms of gear & Sun gear & Planet gear & Gear ring & Number of planetary gear \\
\hline Teeth number of stage 1 & 20 & 40 & 100 & 3 \\
\hline Teeth number of stage 2 & 28 & 36 & 100 & 4 \\
\hline
\end{tabular}

Table 2. Motor speed and the characteristic frequency of the first stage planetary gearbox

\begin{tabular}{|c|c|c|c|c|}
\hline $\begin{array}{c}\text { Motor } \\
\text { speed }\end{array}$ & $\begin{array}{c}\text { Rotating frequency } \\
\text { of sun gear }\left(f_{s}\right)\end{array}$ & $\begin{array}{c}\text { Rotating frequency of } \\
\text { planet gear }\left(f_{p}\right)\end{array}$ & $\begin{array}{c}\text { Rotating frequency of } \\
\text { gear ring }\left(f_{r}\right)\end{array}$ & $\begin{array}{c}\text { Meshing } \\
\text { frequency }\left(f_{m}\right)\end{array}$ \\
\hline $360 \mathrm{r} / \mathrm{min}$ & $6 \mathrm{~Hz}$ & $1 \mathrm{~Hz}$ & $0 \mathrm{~Hz}$ & $100 \mathrm{~Hz}$ \\
\hline
\end{tabular}
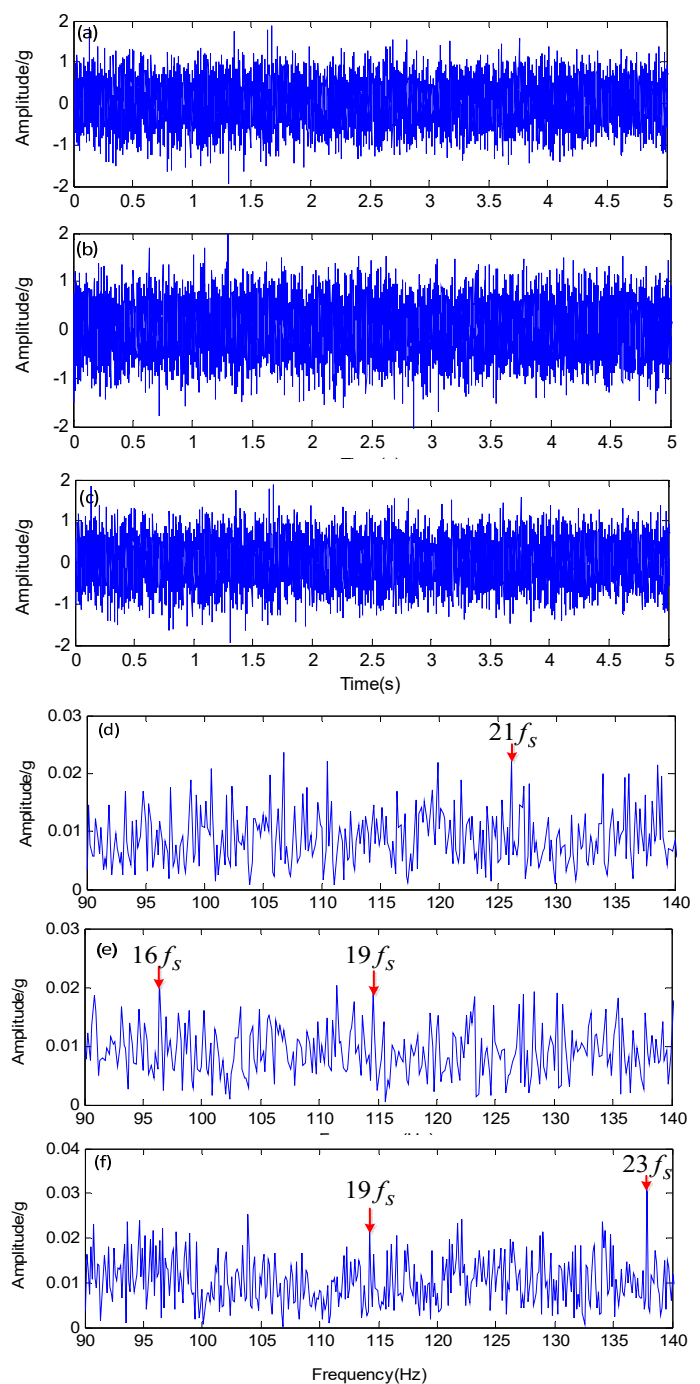

Fig. 9. The time domain of fault signal: a) normal, b) missing tooth, c) broken tooth; The zoom-in spectrum of fault signal: d) normal, e) missing tooth, f) broken tooth

\subsection{Experimental verification}

In the experiment of planetary gearbox, motor rotating frequency is set to $6 \mathrm{~Hz}$, the voltage and electric current of the brake are $1.2 \mathrm{~V}$ and $0.11 \mathrm{~A}$ respectively. The time domain chart of original vibration signal of the first stage sun gear at the states of normal, missing tooth and broken 
tooth are shown in Fig. 9(a)-(c). Due to the strong background noise, it is hardly to distinguish normal signal and periodic impulse signal in the time domain graphs. The zoom-in spectrum diagrams of three states vibration signal are shown in Fig. 9(d)-(f). The sentences have been modified to "In particular, the frequency multiplication of the sun gear is $21 f_{s}$ which appears in zoom-in spectrum of normal state. In zoom-in spectrum of missing tooth graphs, the frequency multiplication of the sun gear are $16 f_{s}$ and $19 f_{s}$. In zoom-in spectrum of broken tooth graphs, the frequency multiplication of the sun gear are $19 f_{s}$ and $23 f_{s}$. In other words, we can see frequency multiplication of the sun gear in the normal and fault signal, without seeing meshing frequency and sidebands. Hence, this cannot be determined whether a failure occurred in the sun gear.
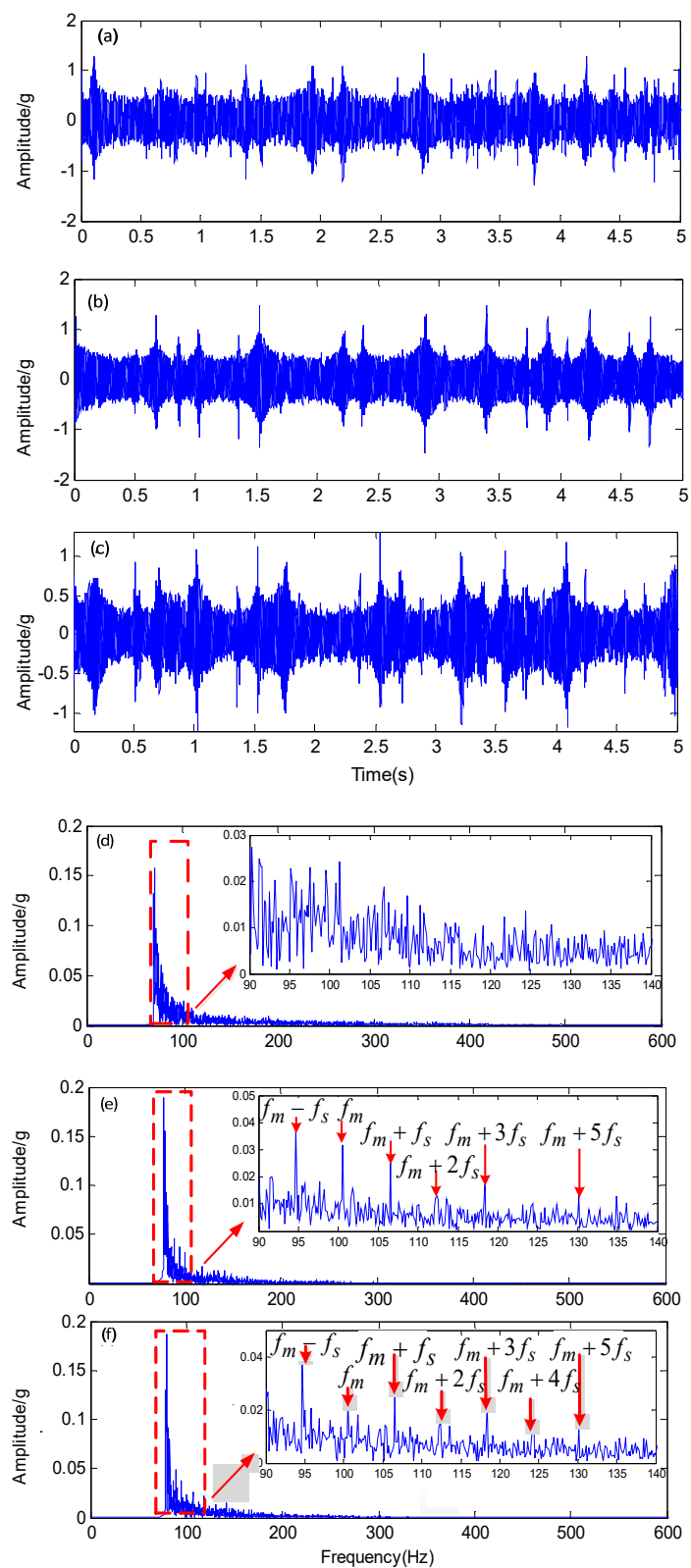

Fig. 10. The time domain of ASR method: a) normal, b) missing tooth, c) broken tooth; The spectrum and zoom-in spectrum of ASR method: a) normal, b) missing tooth, c) broken tooth 
By using the method of proposed in this paper, the vibration signals at the states of normal, missing tooth and broken tooth of the first stage sun gear are processed with frequency-shifted and scaling transform to meet the requirements of small parameters of stochastic resonance. Secondly, the chaos ant colony algorithm optimizes the parameters of the stochastic resonance, and obtain the time domain and frequency spectrum of ASR which are shown in Fig. 10(a)-(f). They are not obvious that the periodic impulse amplitude in Fig. 10(b) and (c) are bigger than that Fig. 10(a). Hence, we cannot judge the sun gear failure corresponding to Fig. 10(b) and (c) happened. In Fig. 10(d), zoomed-in frequency spectrum of the normal vibration signal of the sun gear, periodic impulse peak is not found. However, in Fig. 10(e), the zoomed-in spectrum of the missing tooth, it is easy to find the meshing frequency $f_{m}$ and sidebands appear at $f_{m}-f_{s}$, $f_{m}+f_{s}, f_{m}+2 f_{s}, f_{m}+3 f_{s}, f_{m}+5 f_{s}$. In Fig. 10(f), the zoomed-in frequency spectrum of broken tooth, meshing frequency $f_{m}$ and the sidebands $f_{m}-f_{s}, f_{m}+f_{s}, f_{m}+2 f_{s}, f_{m}+3 f_{s}, f_{m}+4 f_{s}$, $f_{m}+5 f_{s}$ are obtained. Furthermore, from Fig. 10(e) and (f), sidebands interval is the rotating frequency and frequency-doubled of the sun gear. The amplitude of sidebands and meshing frequency are much higher than surrounding noise, which can confirm the sun gear has fault. The analysis results consistent with the actual situation in the experiment. Therefore, the effectiveness of the proposed method is verified by the experiment of planetary gearbox.

For comparison, the singular value decomposition method is used for processing the vibration signal. In Fig. 11(a) and (b) are missing tooth spectrum and broken tooth spectrum of the sun gear. They are clear to see frequency-doubled of the sun gear $6 f_{s}$ in Fig. 11(a), $6 f_{s}$ and $18 f_{s}$ in Fig. 11(b). Because of the interference by strong background noise, the meshing frequency and the sidebands are hidden in the surrounding noise. Thus, the singular value decomposition method cannot be used to extract the fault feature of the weak signal of the planetary gearbox in the strong background noise.
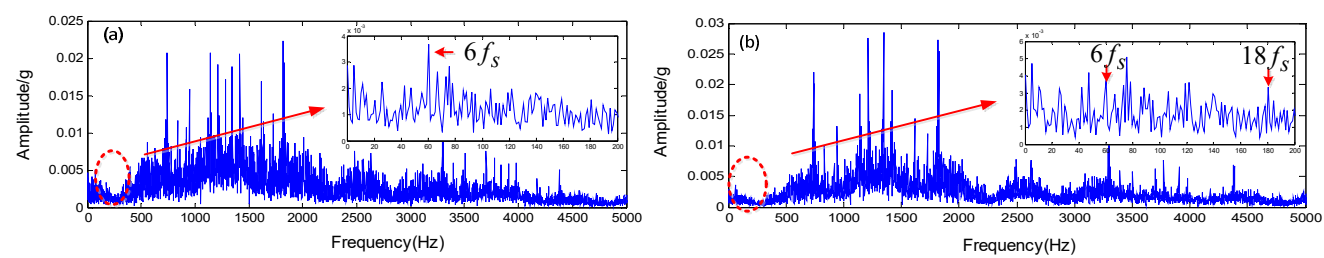

Fig. 11. The spectrum and zoom-in spectrum of singular value decomposition method:

a) missing tooth, b) broken tooth

Through the comparison of the two methods, it can be concluded that, the interaction by interference of strong background noise and complex structure of planetary gearbox.

The fault signal of sun gear is very weak and it is difficult to be extract fault signal by the traditional noise reduction method. The spectrum of the signal has obvious impulse vibration, but the energy of the spectrum is distributed in a high frequency and wide frequency range. In the low frequency, the vibration characteristics are not obvious, and the characteristic frequency of fault is very weak. However, the ASR method can not only reduce the noise but also enhance the weak fault signal. The signal is processed by the adaptive stochastic resonance system, the high frequency noise of signal is filtered out and the useful components of the signal are preserved. For the weak signal is enhanced by the stochastic resonance system, the frequency component of the weak signal is clearly highlighted. This conclusion can be verified in Fig. 5(b), Fig. 10(e) and (f). Therefore, based on the problem of weak fault signal extraction of planetary gearbox, the method proposed in this paper is superior to the singular value decomposition method.

\section{Conclusions}

In this paper, we propose a novel adaptive stochastic resonance method for fault feature extraction in planetary gearbox. The conclusions are as follows: (1) The extraction of weak fault 
feature of planetary gearbox has been a key problem in fault diagnosis. Just reduce noise will inevitably eliminate the useful signal. Stochastic resonance is used to enhance the weak features to achieve effective fault diagnosis. (2) For the deficiency of traditional signal processing methods used in weak signal extraction of planetary gearbox, in this paper, ASR weak signal extraction method is proposed. Chaos perturbation is introduced to overcome the disadvantages of ant colony optimization stochastic resonance (such as slow parameter convergence rate and local optimization), achieves global optimization of system parameters and the optimal stochastic resonance effect. (3) Simulation experiments show that the proposed method can extract weak fault characteristic. In addition, in planetary gearbox test, the vibration signals of the normal, broken tooth and missing tooth of the first stage planetary gearbox were collected, then process the original signal by the proposed method and the singular value decomposition respectively. It can be draw that the proposed method is far superior to the singular value decomposition for weak signal detection of planetary gearbox in strong background noise.

\section{References}

[1] Li H., Zhao J., Song W., Teng H. An offline fault diagnosis method for planetary gearbox based on empirical mode decomposition and adaptive multi-scale morphological gradient filter. Journal of Vibroengineering, Vol. 17, Issue 2, 2015, p. 705-719.

[2] Qiao Z., Lei Y., Lin J., Jia F. An adaptive unsaturated bistable stochastic resonance method and its application in mechanical fault diagnosis. Mechanical Systems and Signal Processing, Vol. 84, Part A, 2017, p. 731-746.

[3] Mosher M. Understanding vibration spectra of planetary gear systems for fault detection. Asme International Design Engineering Technical Conferences and Computers and Information in Engineering Conference, 2003, p. 1-8.

[4] Mcfadden P., Smith J. An explanation for the asymmetry of the modulation sidebands about the tooth meshing frequency in epicyclic gear vibration. Proceedings of the Institution of Mechanical Engineers Part C Journal of Mechanical Engineering Science, Vol. 199, Issue 13, 1985, p. 65-70.

[5] Mcnames J. Fourier series analysis of epicyclic gearbox vibration. Journal of Vibration and Acoustics, Vol. 124, Issue 1, 2002, p. 150-152.

[6] Lei Y., Lin J., Zuo M., He Z. Condition monitoring and fault diagnosis of planetary gearboxes. Journal of Vibration and Control, Vol. 21, Issue 4, 2013, p. 755-764.

[7] Samuel P., Pines D. Constrained adaptive lifting and the CAL4 metric for helicopter transmission diagnostics. Journal of Sound and Vibration, Vol. 319, Issue 1-2, 2009, p. 698-718.

[8] Barszcza T., Randall R. B. Application of spectral kurtosis for detection of a tooth crack in the planetary gear of a wind turbine. Mechanical Systems and Signal Processing, Vol. 23, Issue 4, 2009, p. 1352-1365.

[9] Bai Y., Wang F. Early recognition based on EMD and support vector machine for wind power gear box failure. Industrial Control Computer, Vol. 23, Issue 2, 2010, p. 70-71.

[10] Zhang B., Khawaja T., Patrick R., Vachtsevanos G. Application of blind deconvolution denoising in failure prognosis. IEEE Transactions on Instrumentation and Measurement, Vol. 58, Issue 2, 2009, p. 303-310.

[11] Cheng Z., Hu N., Gao J. Scuffing damage quantitative detection of planetary gear set based on physical model and grey relational analysis. Journal of Vibration Engineering, Vol. 24, Issue 2, 2011, p. 199-204.

[12] Inalpolat M., Kahraman A. A theoretical and experimental investigation of modulation sidebands of planetary gear sets. Journal of Sound and Vibration, Vol. 323, Issue 3-5, 2009, p. 677-696.

[13] Feng Z., Zhao L., Chu F. Vibration spectral characteristics of distributed gear fault of planetary gearboxes. Proceedings of the CSEE, Vol. 33, Issue 2, 2013, p. 118-125.

[14] Feng Z., Chu F. Vibration spectral characteristics of localized gear fault of planetary gearboxes. Proceedings of the CSEE, Vol. 33, Issue 5, 2013, p. 119-127.

[15] Feng Z., Liang M., Zhang Y., Hou S. Fault diagnosis for wind turbine planetary gearboxes via demodulation analysis based on ensemble empirical mode decomposition and energy separation. Renew Energy, Vol. 47, Issue 1, 2012, p. 112-126. 
[16] Feng Z., Zuo M., Qu J., Tian T., Liu Z. Joint amplitude and frequency demodulation analysis based on local mean decomposition for fault diagnosis of planetary gearboxes. Mechanical Systems and Signal Processing, Vol. 40, Issue 1, 2013, p. 56-75.

[17] Mark W. Stationary transducer response to planetary-gear vibration excitation II: effects of torque modulations. Mechanical Systems and Signal Processing, Vol. 23, Issue 7, 2009, p. 2253-2259.

[18] Mark W., Hines J. Stationary transducer response to planetary-gear vibration excitation with non-uniform planet loading. Mechanical Systems and Signal Processing, Vol. 23, Issue 4, 2009, p. 1366-1381.

[19] Benzi R., Sutera A., Vulpiana A. The mechanism of stochastic resonance. Journal of Physics A: Mathematical and General, Vol. 14, Issue 11, 1981, p. L453-L457.

[20] Shi P., Ding X., Han D. Study on multi-frequency weak signal detection method based on stochastic resonance tuning by multi-scale noise. Measurement, Vol. 47, Issue 1, 2014, p. 540-546.

[21] Lei Y., Han D., Lin J., He Z. Planetary gearbox fault diagnosis using an adaptive stochastic resonance method. Mechanical Systems and Signal Processing, Vol. 38, Issue 1, 2013, p. 113-124.

[22] Lu S., He Q., Zhang H., Kong F. Rotating machine fault diagnosis through enhanced stochastic resonance by full-wave signal construction. Mechanical Systems and Signal Processing, Vol. 85, 2017, p. 82-97.

[23] Lu S., He Q., Kong F. Stochastic resonance with Woods-Saxon potential for rolling element bearing fault diagnosis. Mechanical Systems and Signal Processing, Vol. 45, Issue 2, 2014, p. 488-503.

[24] Chen X., Cheng G., Shan X., Hu X., Guo Q, Liu H. Research of weak fault feature information extraction of planetary gear based on ensemble empirical mode decomposition and adaptive stochastic resonance. Measurement, Vol. 73, Issue 9, 2015, p. 55-67.

[25] Liu X., Yang J., Liu H., Cheng G., Chen X., Xu D. Optimizing the adaptive stochastic resonance and its application in fault diagnosis. Fluctuation and Noise Letters, Vol. 14, Issue 4, 2015, p. 1550038.

[26] Qiao Z, Lei Y., Lin J. Stochastic resonance subject to multiplicative and additive noise: the influence of potential asymmetries. Physical Review E, Vol. 94, Issue 5, 2016, p. 052214.

[27] Randall R. B., Antoni J. Rolling element bearing diagnostics-a tutorial. Mechanical Systems and Signal Processing, Vol. 25, Issue 2, 2011, p. 485-520.

[28] Qiao Z., Pan Z. SVD principle analysis and fault diagnosis for bearing based on the correlation coefficient. Measurement Science and Technology, Vol. 26, Issue 8, 2015, p. 085014.

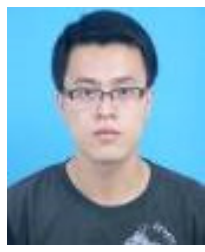

Zhixing Li received M.S degree in School of Mechanical Engineering Institute from University of Science and Technology Lnner Mongolia, Baotou, People's Republic of China, in 2011. Now he is Ph.D. candidate at University of Science and Technology Beijing. His current research interests include vibration detection and fault diagnosis.

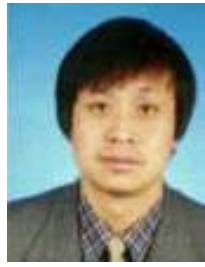

Boqiang Shi received Ph.D. degree in Mining Machinery Institute from University of Science and Technology Beijing, Beijing, People's Republic of China, in 1994. Now he works at University of Science and Technology Beijing. His current research interests include Special vehicle design, condition monitoring and fault diagnosis of mechanical system. 